\title{
The Data-Driven Product-Service Systems Design and Delivery (4DPSS) Methodology
}

\author{
Roberto Sala ${ }^{1(凶)}(\mathbb{D})$, Alessandro Bertoni ${ }^{2}$ (D), Fabiana Pirola ${ }^{1}$ (D), \\ and Giuditta Pezzotta ${ }^{1}$ (D) \\ 1 Department of Management, Information and Production Engineering, \\ University of Bergamo, Viale Marconi, 5, 24044 Dalmine, BG, Italy \\ roberto.sala@unibg.it \\ 2 Department of Mechanical Engineering, Blekinge Institute of Technology, \\ 37179 Karlskrona, Sweden
}

\begin{abstract}
The design of Product-Service Systems (PSS) has been approached from several perspectives like the process, innovation, engineering and operational ones providing, for each one of those, a specific view on the problem. This paper proposes a Data-Driven Product-Service System Design and Delivery (4DPSS) methodology focusing on the collection and exploitation of delivery data to feed the design phase. The logic of the methodology relies on aggregating operational data (collected in the delivery phase) to build a consistent body of knowledge to be exploited in iterative PSS design activities, thanks to better identification of customer needs, and product and service process design issues. This paper presents the 4DPSS methodology at a theoretical level, the implementations of the different methods constituting the methodology are referred to in the text, while its implementation and test as a whole are demanded to future work.
\end{abstract}

Keywords: Product-service system $\cdot$ Data-driven design $\cdot$ Data-driven delivery

\section{Introduction}

Companies approaching the transition towards the offering of Product-Service Systems (PSS) face challenges encompassing the definition of a successful business model, the design of the efficient and sustainable product and service combinations, and the definition of operational procedures supporting the effective PSS delivery. A plethora of methods to support the transition toward PSS has been proposed in the literature. Such methods, despite approaching the challenge from different perspectives, have as a common denominator the capability to correctly capture and fulfil the needs of the customers, and use them to guide the whole PSS design [1] and delivery process [2].

The shift from a traditional product-centric perspective to the PSS perspective has brought researchers and community of practitioners to focus on the multi-disciplinary implications of such a new mindset. The "PSS transition" challenge has been approached from a process perspective, focusing on lean processes and waste reduction in service and maintenance integrating operational data [3, 4], from a PSS innovation perspective, focusing on design space exploration and value and sustainability 
assessment enabled by surrogate modelling [5], and from an engineering practice perspective, focusing on modelling and visualization to address the cognitive limitation of engineers' when making decisions in the presence of multi-disciplinary information [6]. Another factor that should be considered as influencing the transition towards PSS is the digitalization of companies, which can be beneficial in terms of data availability for design and delivery purposes [7]. All of these perspectives have been addressed singularly, without considering their integration. Thus, the paper proposes a methodology that, having roots in methodologies and tools presented in the literature, integrates the different perspectives to improve the PSS design and delivery. In the following sections, the paper introduces the main theoretical foundation of the methodology, explain the research approach, and describe and visualize the details of the proposed methodology.

\section{Scientific Positioning}

\subsection{Theoretical Foundation}

As an answer to need to provide guidance addressing such different dimensions, this paper presents a methodology, named Data-Driven Product-Service Systems Design and Delivery (4DPSS), developed to support the deployment of PSS solutions by creating a virtuous circle of data and information sharing between the PSS design phase and the PSS operations. In particular, the uniqueness of the methodology resides in the adoption of a four-dimensional perspective addressing both the PSS design and the PSS operations during delivery. Figure 1 illustrates such perspectives, that consists in: (1) dealing with the process-oriented view optimizing the design process phases (i.e. the design perspective), (2) assessing the fulfilment of multi-disciplinary and multidimensional needs of the different stakeholders, encompassing lifecycle-value and sustainability dimensions (i.e. the value modelling perspective), (3) dealing with the engineering-human dimension of the design activity, introducing the use of boundary objects to nurture discussion and understanding of the multi-disciplinary implications of PSS design decisions (i.e. the PSS visualization perspective), (4) integrating the design process with data from the field, collected during the delivery phase as support for needs identification and effective PSS design (i.e. operational perspective).

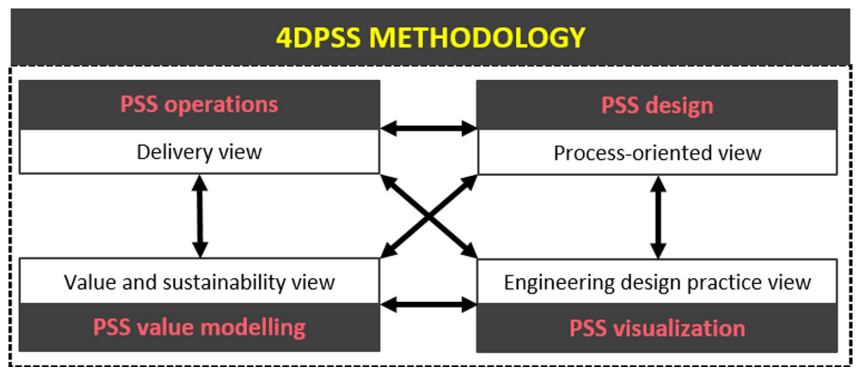

Fig. 1. Four-dimensional perspective of the 4DPSS methodology 
Different research contributions address such perspectives one at a time. The proposed methodology builds on some of the main approaches proposed in PSS research, particularly focusing on how the increasing availability of information communication technologies, combined with data science, can enable an effective and efficient PSS deployment. From a process-oriented perspective the PSS Lean Design Methodology (PSSLDM) described in [3], proposes a comprehensive approach to develop PSS along the entire lifecycle, defined by four main phases: the identification of the customer needs, the PSS conceptual design, the PSS design and the monitoring of the PSS performance when on the market. The PSSLDM introduces the adoption of specific KPIs to monitor PSS performances on the market, to facilitate the identification of PSS problems [8].

The iterative use and evolution of value models along the PSS design process have been proposed $[5,6]$. Such an iterative process is enabled by the use of surrogate modelling and data mining to explore a wider design space [6]. This triggers design negotiations, forcing the PSS cross-functional team members to "(1) confront each other's perceptions on what the value of a system is, to (2) resolve conflicts where conclusions differ, and to (3) progressively learn what a 'good design' is" [3, p. 18]. To populate such models with reliable information, researchers have investigated how models should be built based on data collected from product operations, historical databases, sensors, and online resources, and how unexpected correlations can be discovered by the use of data mining and machine learning [9]. The effectiveness of using such value models to frontload engineering design activities is strongly related to their effectiveness in enhancing cross-disciplinary and cross-functional collaboration in early design, in other words to their ability to act as effective boundary objects in decision making. This has ultimately led to the proposal of a physical interactive visualization environment and interfaces to enhance the multidisciplinary awareness of decision-making teams when dealing with ill-defined design problems encompassing both a product and service dimensions [6].

From the delivery perspective, the 4DPSS methodology integrates the operational data gatherable during the PSS delivery (e.g. during maintenance services) to improve the recognition of problems at the design or process level. This activity is supported through the implementation of a structured approach, as presented in [4], enhancing the data collection, management and analysis, which are three central aspects for value generation from data [10].

\subsection{Research Approach}

The definition of the research questions and objectives at the basis of the paper is the results of a research clarification activity based on an extensive literature review in the field of PSS design and delivery, combined with a participatory action research approach in several industrial contexts. The authors have directly participated in industrial research initiatives aiming to facilitate the design and operationalization of PSS, as well as contributing to the development of case studies on the topic. Four Swedish medium-large companies in the aerospace, construction machining, and packaging industry have been involved in the investigation of needs in terms of PSS concept design and trade-off, regarding mainly the current PSS design process in terms 
of phases and data used for decision-making purposes. Similarly, three Italian manufacturing companies, producing bottling and packaging machines, balancing machines and pumps for the Oil\&Gas sector, have been engaged to support the definition of the operational characteristics supporting the collection of information to be used later in the design phase. For these interviews, the attention was devoted to the data generated during the maintenance delivery and used for maintenance decision-making (e.g. technician selection and intervention scheduling).

Qualitative data were gathered through semi-structured interviews, workshops and focus groups, initially to compile a comprehensive descriptive analysis of the needs and challenges of PSS design and delivery, and later with the intent to preliminary validate the different steps of the methodology.

\section{The 4DPSS Methodology}

This section deals with the description of the 4DPSS methodology structure. As introduced in Sect. 1, the paper proposed a methodology covering different aspects of the PSS design and delivery, integrating different perspectives and working on different levels, from the process one (definition of the main phases) to the operative one (definition of the sub-phases and the decision-making tools).

The methodology describes how the integration of different perspectives can be concretized by making the best use of the data collection and analysis in the PSS design, implementation and monitoring phases. Figure 1 illustrates visually the fourdimensional perspective adopted by the 4DPSS methodology, encompassing the focus on PSS design, on value modelling for design space exploration and trade-off, visualization for engineering design practice and decision making, and the integration of data from the field.

The integration of the four perspectives described in Fig. 1 results into the DataDriven Product-Service Systems Design and Delivery (4DPSS) methodology (Fig. 2). Each one of the perspectives contributes in supporting the phases of the 4DPSS by providing different views on the design (e.g. PSS design, PSs value modelling and PSS visualization) and delivery problems (e.g. PSS operations).

The 4DPSS is composed of four consecutive phases: i) Needs identification, ii) Concept generation and design, iii) Implementation and, iv) Monitoring. Each phase can be characterized by one or more sub-phases to carry out specific tasks.

The first phase is characterized by the identification of the needs leading to the conceptualization of the PSS characteristics and components. In particular, needs can stem from three different sources: a) customer, b) product and, c) service. The customers' needs originate from the direct dialogue with the customer and are collected through different means such as interviews, surveys or analysis of customer feedback on social platforms. 


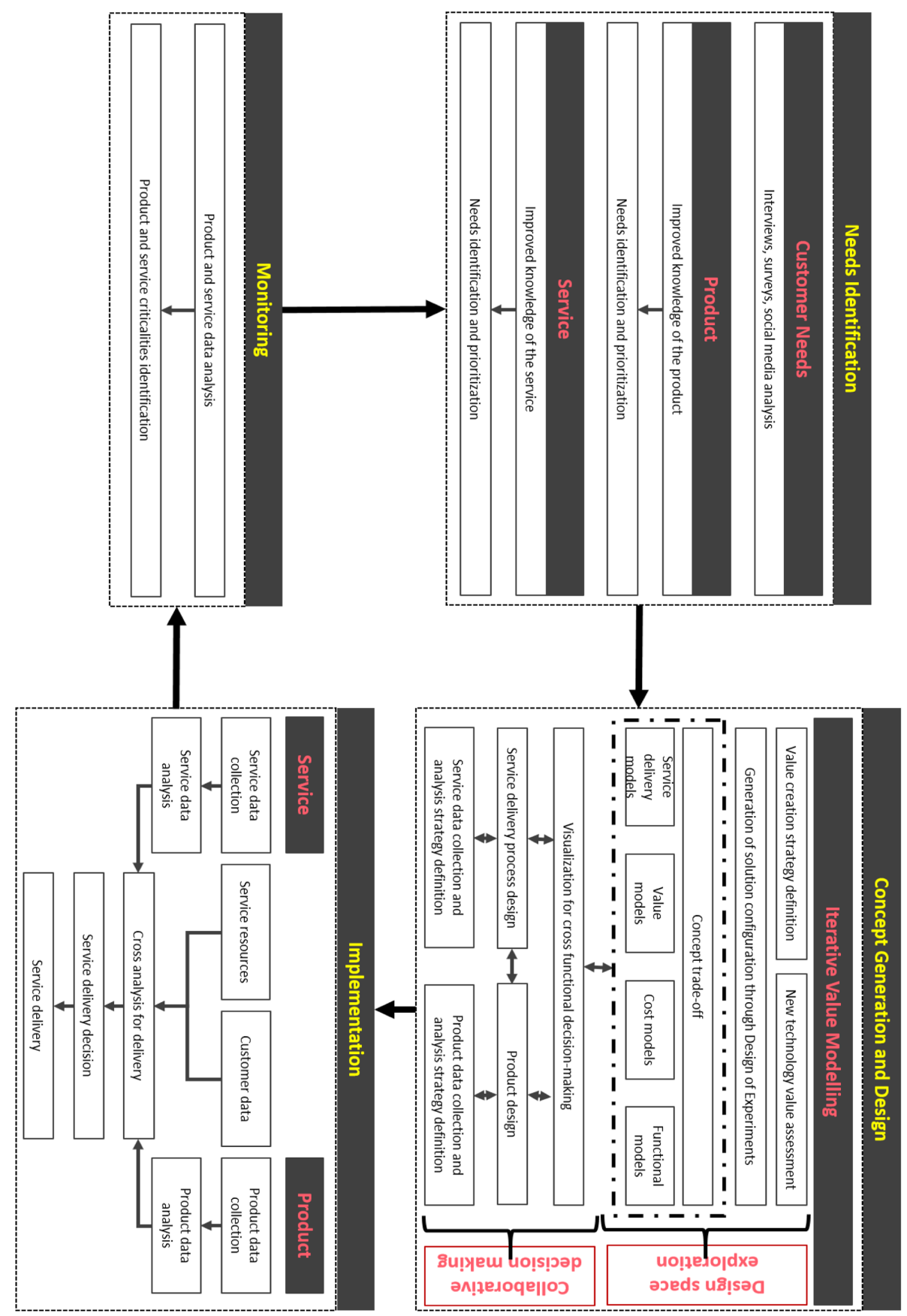

Fig. 2. The 4DPSS methodology 
Instead, the product and service needs are the results of improved knowledge of the product and service thanks to the data analysis and criticalities identification activities carried out in the Monitoring phase.

The prioritization of the needs is performed according to the criticality of the problem identified, usually associated with the time or cost required to solve the problem.

The second phase, Concept generation and design, uses the information collected in the previous phase as inputs for the creation of PSS concepts. The Value Creation Strategy is updated based on the data collected in the needs identification phase, and it is used to assess the potential value of new technologies. At this stage, a number of potential design modifications of a baseline design are decided and the design space is explored by running simulation models. In case of complex and critical product design, where a set of simulations can take up to days or weeks, such as in aerospace component design, a Design of Experiment setting can be used to generate a large enough set of simulation results to create surrogate models approximating the whole design space (see [6] for more details).

Such information converges in a collaborative decision-making environment, featuring visualization capabilities, where models are used as boundary objects to enhance engineers' awareness decision making. In parallel with the exploration of alternative product design, the PSS design activities are also characterized by the definition of specific data collection and analysis strategies for the service and the product components, as suggested in [4]. The definition of proper data-related strategies is fundamental to enable profitable PSS delivery and to understand what kind of information could be collected and analyzed during the use phase to improve service delivery.

The third phase, Implementation, is focused on the collection of the data from the field supporting the decision-making phase at the operational level. The effectiveness and operational efficiency of this phase are subjected to the decisions made in the previous phase. This phase should support the operational decision making exploiting historical and aggregated data collected during the PSS delivery. Data sources are different and cover both product and service sides, involving also customers as long as their general information is involved. Data collection during PSS use is aimed at creating a comprehensive database supporting the decision-making phase in service delivery [4]. The cross-analysis and the service delivery decision could be supported by tools such as optimization models able to consider product and service information.

The fourth phase, Monitoring, exploits the aggregated data collected in the Implementation phase to extract information concerning the PSS performance to identify problems and criticalities. In particular, analyses are focused both on the product and service-related data. For example, the analysis of maintenance data could be used to identify problems in the product design (e.g. breakdowns and stoppages due to the frequent failure of the same component), or in the way service delivery is designed (e.g. useless activities in the process, wrong tools). Thus, this phase aims to extract information that could be used as input for the following phase, Needs identification. With the proposed approach, needs won't be identified only through the experience of the designer or the direct inputs of the customers, but also through a structured analysis of the data collected from the PSS on the field. 
The 4DPSS methodology finds its roots in methodologies and methods discussed in the literature. The innovativeness and the value-added, of the 4DPSS stems from the integration of these methods and methodologies, developed to reduce the wastes along with the PSS design and delivery phases, and improve the effectiveness of the PSS delivery through data-driven design and delivery. Methods and methodologies have been selected due to their synergies concerning the inputs and outputs of each phase in the scope of improving the PSS design and increase the effectiveness of the need identification phase enlarging the pool of sources not only to feedback from customers but considering also the data from the service delivery phase.

\section{Discussion and Conclusions}

The 4DPSS methodology introduces a data-driven decision-making approach exploiting aggregated operational data to identify needs and emerging PSS criticalities from the operational phase and use them in both PSS design and delivery. The 4DPSS methodology shall be seen as a bridge between the literature focusing on PSS development, PSS innovation and design exploration, and PSS visualization for decisionmaking. The necessity for such a methodology finds its root in the lacks identified through interviews and collaboration with the companies. Specifically, it emerged the incoherence between data collection and exploitation approaches as they are currently carried out in the companies, mainly due to the problems in data management and analysis for design and delivery purposes. For example, some of the interviewed companies had experience with the data-driven design methods part of the "Concept generation and design" phase, but no experience with what is proposed in the "Implementation" phase. Similarly, other companies were experienced with the "Implementation" and "Monitoring" phases but had no experience with the methods proposed in the "Concept generation and design" phase. In this sense, it emerged the interest from companies in introducing digital supports and perspectives able to improve the internal processes related to the design phase and then, improve the ones related to the service delivery [7]. Thus, the research approach allowed identifying the blind spots related to data utilization in the various phases and propose solutions to tackle them through a mix of knowledge taken from literature, company experience and researchers' experience.

The design, implementation, and monitoring of PSS rely on a data-driven approach that works both at the operational and strategic levels and that is aimed at improving, (1) the maintenance delivery through improved operational decisions, (2) the PSS composition through an analysis of the components who fails most frequently and of the service delivery process characteristics, identifying and correcting the most critical activities, (3) the PSS design process by populating value models with a constant flow of information derived from the analysis of the data collected during the PSS operations.

Finally, the proposed methodology should be considered as the results of a prescriptive study and it has not been implemented and validated in its entirety in a real case study. Nevertheless, the methods and approaches proposed in its frame have been singularly validated in the different research initiatives cited in the paper. Future work 
will focus on the implementation and test of the methodology in a single case study. The validation process of the 4DPSS methodology would require to apply it in its entirety to a single case study to evaluate its effectiveness and the implementation barriers. Given the long time required to collect operational data for new PSS, the identification of needs from operational data will require to identify a company who is already collecting operational data from maintenance in a suitable way, which allows applying the logic part of the 4DPSS.

\section{References}

1. Maussang, N., Zwolinski, P., Brissaud, D.: Product-service system design methodology: from the PSS architecture design to the products specifications. J. Eng. Des. 20, 349-366 (2009)

2. Tian, G., Zhang, H., Zhou, M., Li, Z.: AHP, gray correlation, and TOPSIS combined approach to green performance evaluation of design alternatives. IEEE Trans. Syst. Man Cybern. Syst. 48, 1093-1105 (2018)

3. Pezzotta, G., et al.: The product service system lean design methodology (PSSLDM): integrating product and service components along the whole PSS lifecycle. J. Manuf. Technol. Manag. 29, 1270-1295 (2018)

4. Sala, R., Pirola, F., Dovere, E., Cavalieri, S.: A dual perspective workflow to improve data collection for maintenance delivery: an industrial case study. In: Ameri, F., Stecke, K.E., von Cieminski, G., Kiritsis, D. (eds.) APMS 2019. IAICT, vol. 566, pp. 485-492. Springer, Cham (2019). https://doi.org/10.1007/978-3-030-30000-5_60

5. Bertoni, M., Bertoni, A.: Iterative value models generation in the engineering design process. Des. Sci. 5 (2019)

6. Bertoni, M., Wall, J., Bertoni, A.: Model Driven Decision Arena: an areospace study. In: Proceedings of the International Design Conference, pp. 171-182 (2018)

7. Ardolino, M., Rapaccini, M., Saccani, N., Gaiardelli, P., Crespi, G., Ruggeri, C.: The role of digital technologies for the service transformation of industrial companies. Int. J. Prod. Res. 1-17 (2017)

8. Mourtzis, D., Papatheodorou, A.-M., Fotia, S.: Development of a Key Performance Indicator Assessment Methodology and Software Tool for Product-Service System Evaluation and Decision-Making Support (2018)

9. Bertoni, A., Bertoni, M.: PSS cost engineering: a model-based approach for concept design. CIRP J. Manuf. Sci. Technol. (2018)

10. Zhou, J., Yao, X., Zhang, J.: Big data in wisdom manufacturing for industry 4.0. In: 2017 5th International Conference Enterprise Systems, pp. 107-112 (2017) 